\title{
Circulating endothelial progenitor cells in multiple myeloma: implications and significance
}

Hong Zhang, Varsha Vakil, Marc Braunstein, Eric L. P. Smith, Justin Maroney, Laurie Chen, Kezhi Dai, James R. Berenson, M. Mahmood Hussain, Uwe Klueppelberg, Allen J. Norin, Hasan O. Akman, Tayfun Özçelik, and Olcay A. Batuman

\begin{abstract}
Angiogenesis governs the progression of multiple myeloma (MM). Circulating endothelial cells (CECs) contribute to angiogenesis and comprise mature ECs and endothelial progenitor cells (EPCs). The present study sought to characterize CECs and their relation to disease activity and therapeutic response in 31 consecutive patients with MM. CECs, identified as CD34 ${ }^{+} / \mathrm{CD} 146^{+} / \mathrm{CD}_{105}{ }^{+} / \mathrm{CD} 11 \mathrm{~b}^{-}$cells, were 6-fold higher in patients compared to controls and correlated positively with serum $M$ protein and $\boldsymbol{\beta}_{2}$-microglobulin. Circulating EPCs displayed late colony
\end{abstract}

formation/outgrowth and capillary-like network formation on matrigel; these processes were inhibited after effective thalidomide treatment. Co-expression of vascular endothelial growth factor receptor-2 (KDR) and CD133 characterized EPCs in MM, and KDR mRNA elevations correlated with $M$ protein levels. In vitro exposure of ECs to thalidomide or its derivative CC-5013 inhibited gene expression of the receptors for transforming growth factor- $\beta$ and thrombin. Thus, elevated levels of CECs and EPCs covary with disease activity and re- sponse to thalidomide, underscoring the angiogenic aspect of $\mathrm{MM}$ and suggesting that angioblastlike EPCs are a pathogenic biomarker and a rational treatment target in MM. The results also highlight the anti-angiogenic properties of thalidomide and CC-5013 and further elucidate possible mechanisms of their effectiveness against MM. (Blood. 2005;105: 3286-3294)

() 2005 by The American Society of Hematology

\section{Introduction}

In multiple myeloma (MM), the growth of neoplastic plasma cells is directly regulated by microenvironmental factors in the bone marrow. ${ }^{1}$ Of these, neoangiogenesis is thought to have a governing role in MM pathogenesis and progression, indicated by increased bone marrow microvascular density (MVD) in patients that is positively correlated with disease activity and by increased expression of angiogenic factors. ${ }^{2-6}$ In fact, increased MVD in myeloma is thought to be largely driven by vascular endothelial growth factor (VEGF), which is secreted by MM and stromal cells in the marrow; these cells also express the receptors for VEGF, including VEGF receptor-2 (kinase insert domain-containing receptor/fetal liver kinase-1 [KDR])..$^{7-9}$ The production of VEGF by both cell types is increased by interleukin-6 (IL-6), which is produced by stromal cells and provides the survival signal for MM cells; VEGF in turn stimulates production of IL-6 and tumor growth. ${ }^{10}$

Targeting of angiogenesis by thalidomide ${ }^{11-14}$ and its more potent immunomodulatory derivatives (IMiDs; Celgene, Warren, $\mathrm{NJ})^{15,16}$ is an effective therapeutic strategy against MM in newly diagnosed, relapsed, and refractory patients. Recent data showing a proangiogenic gene expression profile within bone marrow endothelial cells (ECs) of patients with MM suggest that these ECs had undergone an angiogenic switch ${ }^{17}$ similar to that observed in solid

From the Division of Hematology/Oncology, the Department of Medicine; the Department of Anatomy and Cell Biology, State University of New York Downstate Medical Center, Brooklyn, NY; the Institute for Myeloma \& Bone Cancer Research, University of California at Los Angeles (UCLA) School of Medicine, Los Angeles, CA; the Ayhan Sahenk Foundation and Bilkent University, Department of Molecular Biology and Genetics, Bilkent, Ankara, Turkey; the Transplant Immunology \& Immunogenetics Laboratory, State University of New York Downstate Medical Center, Brooklyn, NY.

Submitted June 4, 2004; accepted December 15, 2004. Prepublished online as Blood First Edition Paper, December 23, 2004; DOI 10.1182/blood-200406-2101. tumors. ${ }^{18,19}$ However, the role of neovascularization in mechanisms involved in MM growth and dissemination requires further elucidation. From the clinical perspective, since MVD may not reflect real-time vascular activity in the marrow, and since MVD determination requires a bone marrow biopsy, a less invasive method that can assess dynamic changes in angiogenesis is needed for gaining insight into the natural course of $\mathrm{MM}$ and improving patient management.

Growth and dissemination of tumors is supported by neovascularization (new vessel formation), which involves vasculogenesis (de novo tube formation analogous to prenatal vascular development) and angiogenesis (sprouting of new capillary vessels from pre-existing vasculature). Exciting new data show that tumor neovascularization involves recruitment of endothelial progenitor cells (EPCs) from bone marrow and, conversely, that inhibition of EPC recruitment inhibits tumor growth, thus establishing the significance of EPCs in tumor progression. ${ }^{20-24}$ Consequently, inhibition of EPCs is a promising treatment modality with benefit both for solid and for liquid tumors. New research also has shown that within human bone marrow and cord blood, EPCs are derived from pluripotential stem cells and from more differentiated hemangioblasts, which are the precursors for both hematopoietic cells and

Supported by a Multiple Myeloma Research Foundation Senior Research Award (O.A.B.) and by research funding from Celgene (O.A.B.).

An Inside Blood analysis of this article appears in the front of this issue.

Reprints: Olcay A. Batuman, Division of Hematology/Oncology, Department of Medicine, SUNY Downstate Medical Center, Box 20, 450 Clarkson Ave, Brooklyn, NY 11203; e-mail: obatuman@downstate.edu.

The publication costs of this article were defrayed in part by page charge payment. Therefore, and solely to indicate this fact, this article is hereby marked "advertisement" in accordance with 18 U.S.C. section 1734.

(C) 2005 by The American Society of Hematology 
ECs. ${ }^{21,25-29}$ At steady state, $0.01 \%$ of cells in circulation can be identified as EPCs; the remaining CECs consist of mature EC populations. ${ }^{21}$ As distinct from mature CECs, EPCs in circulation form late colonies in vitro that expand into EC monolayers, migrate into tumor tissue in vivo, grow into 3-dimensional cultures in vitro, and have a high proliferative rate. ${ }^{22,25,26,30-32}$ These properties suggest that EPCs resemble embryonic angioblasts, which characteristically migrate, proliferate, and differentiate into mature ECs capable of vasculogenesis. ${ }^{33,34}$ Differentiated ECs and EPCs cannot be distinguished by phenotypic characteristics alone, because they express similar endothelial antigens that are also expressed by other hematopoietic cells. In general, EPCs can be identified by simultaneous expression of the cell surface markers CD34, ${ }^{30,35}$ CD133 (AC133), ${ }^{31}$ and KDR. ${ }^{24}$ Increased mobilization of CECs and EPCs has been associated with cancer, vascular injury and, in patients with lymphoma, poor prognosis. ${ }^{36-38}$

In MM, EPCs in peripheral blood have not been characterized, and their role in disease pathogenesis and progression are unknown. In the current study, we tested the hypothesis that the level and function of EPCs in MM correlate with disease activity. Data from 31 consecutive patients show that levels of CECs and angioblastlike EPCs in peripheral blood are higher in patients than in healthy controls. The frequency of CECs and EPCs in patients was responsive to effective treatment with thalidomide and also positively correlated with levels of serum $M$ protein and $\beta_{2^{-}}$ microglobulin, which indicate disease activity in MM. In vitro studies that explored mechanisms of actions of thalidomide on ECs showed that both thalidomide and its immunoregulatory derivative (IMiD) CC-5013 (Revlimid; Celgene) decreased mRNA levels of endothelial genes that regulate vasculogenesis and angiogenesis, including those for ser/thr kinase receptor I for the cytokine transforming growth factor- $\beta$ (TGF- $\beta$ ), which regulates vasculogenesis and tumor growth, ${ }^{39,40}$ and thrombin receptor (TR), a cell invasion protease that is expressed at higher levels by MM cells compared to normal plasma cells. ${ }^{41}$

Taken together, these results underscore the vascular endothelial aspect of MM and suggest that EPCs in peripheral blood play an important role in MM's severity and that their decrease is associated with response to thalidomide. Furthermore, the current results indicate that circulating EPCs are a new treatment target in MM and suggest that EPCs may serve as a readily accessible biomarker of disease status.

\section{Patients, materials, and methods}

\section{Patients}

Thirty-one consecutive nonrelapsing patients with MM followed at Downstate Medical Center's Hematology Clinic served as subjects. All fulfilled the Southwest Oncology Group (SWOG) diagnostic criteria for MM. ${ }^{42}$ Approval was obtained from the State University of New York (SUNY) Downstate and Kings County Hospitals' Institutional Review Boards, and informed consent was obtained in accordance with the Declaration of Helsinki. Indicated numbers of patients as noted in Figures 1 through 6 had the described studies.

\section{Characterization of circulating endothelial cells}

Cytometric analysis. Heparinized peripheral blood $(20 \mathrm{~mL})$ was obtained from patients and from controls, who were healthy hospital staff. Peripheral blood mononuclear cell (PBMC) layer was separated by Ficoll-Hypaque (Sigma Chemicals, St Louis, MO) density-gradient centrifugation within 6 hours of blood collection. Paired samples from patients and controls were analyzed by 3-color flow cytometry using combinations of monoclonal antibodies (mAbs) obtained from BD Biosciences-PharMingen (San Diego, $\mathrm{CA}$ ): anti-CD34-phycoerythrin (PE); anti-CD105 conjugated to fluorescein isothiocyanate (FITC) using the FluoroTag FITC conjugation kit (Sigma Chemicals) according to manufacturer's instructions; CD11b-CyChrome; anti-CD146-PE; anti-CD34-FITC; and anti-CD38-FITC. Briefly, direct immunofluorescence staining was performed by incubation of $1 \times 10^{6}$ cells with $20 \mu \mathrm{L}$ of $\mathrm{mAb}$ for 30 minutes at $4^{\circ} \mathrm{C}$. To ensure detection of low levels of ECs, at least 100000 cells from each sample were acquired; data were analyzed with a FACSort flow cytometer (BD Biosciences PharMingen) using the CellQuest software program (BD Immunocytometry Systems, San Diego, CA). To assess reproducibility, CEC frequency in all subjects was determined at least twice in 2 separate blood samples obtained 1 to 3 days apart.

Endothelial progenitor cell colony-forming assay and culture. To assess the frequency of EPCs in patients with MM, PBMCs were resuspended in medium 199 (Gibco BRL, Grand Island, NY) supplemented with endothelial cell growth supplement $(50 \mu \mathrm{g} / \mathrm{mL}$; Calbiochem, San Diego, CA), 20\% fetal bovine serum, antibiotics (100 U/mL penicillin $\mathrm{G}, 100 \mu \mathrm{g} / \mathrm{mL}$ streptomycin), and heparin $(50 \mu \mathrm{g} / \mathrm{mL}$, porcine intestinal; Sigma Chemicals), and were cultured in duplicate in fibronectin-coated 6-well plates (Becton Dickinson Labware, Bedford, MA). To eliminate contamination of the assay by mature CECs, nonadherent cells were collected after 48 hours and replated onto laminin-coated 6-well plates (Becton Dickinson Labware). Medium was changed every 3 days, and the number of colonies was counted 14 to 18 days after plating. EC colonies were defined in the manner of Hill et $\mathrm{al}^{37}$ as consisting of multiple thin, flat cells emerging from a cluster of round cells. Colonies were quantitated in a minimum of 6 fields (original $\times 40$ ) per well by 2 observers without knowledge of the subjects' clinical profiles (colony forming unit [CFU]-EPC score). To assess reproducibility and to confirm endothelial origin of the outgrown cells, nonadherent cells from fibronectin-coated 6-well plates were also placed into laminin-coated 96-well plates (Becton Dickinson Labware) for analysis by immunocytochemistry. The correlation between CFU counts of laminin-coated 6-well and 96-well cultures was 0.92 .

Capillary-like network formation on matrigel. Aliquots $(200 \mu \mathrm{L})$ of $0.1 \%$ matrigel (BD Biosciences) were added to the wells of precooled 48-well culture plates and were incubated at $37^{\circ} \mathrm{C}$ until gelation occurred. Capillary tube formation after 14 days of culture was evaluated on a scale based on percent coverage of the well surface of the plate using an inverted Nikon Diaphot 300 microscope equipped with a grid (Nikon, Melville, NY). Anastomosing capillary-like networks that covered more than $90 \%$ of the base of the well were graded as +3 ; expansion to $50 \%$ to $90 \%$ was graded as $+2 ; 25 \%$ to $50 \%$ was graded as +1 ; and less than $25 \%$ was graded as 0 . The mean of triplicate wells from each patient at indicated times determined their score.

\section{Immunocytochemistry}

Confirmation of endothelial lineage was performed by indirect immunostaining with endothelial-specific mAbs directed against KDR, CD144 (vascular endothelial cadherin [VE-cadherin]), and von Willebrand factor (VWF); anti-CD38 was also used to detect circulating MM cells. Briefly, adherent cells in $96-$ well plates were fixed in $4 \%$ paraformaldehyde in $1 \times$ phosphate-buffered saline, $\mathrm{pH} 7.2$, containing $0.02 \%$ sodium azide; treated with $0.05 \mathrm{M}$ glycine; and permeabilized with Triton X-100. Blocking with 5\% serum was followed by overnight incubation with primary antibody at $4{ }^{\circ} \mathrm{C}$ and secondary antibody goat-anti-mouse Alexa Fluor 488 or 568 (Molecular Probes, Eugene, OR). For 2-color studies, following staining with the first antibody, staining with a second primary antibody was done after blocking with normal serum. Appropriate isotype-matched serum with each of the primary antibodies was used as a negative control. Images of the stained cells were digitally recorded in sequential mode on a confocal laser scanning microscope (Bio-Rad MRC 1024ES; Bio-Rad, Hercules, CA). 


\section{Real-time quantitative reverse transcription-polymerase chain reaction (RT-PCR) analysis for KDR and VE-CADHERIN}

The one-step RT-PCR analysis was performed on an ABI PRISM 7000 sequence detector (Applied Biosystems, Foster City, CA) according to the manufacturer's instructions. After treatment with RNase-free DNase (Invitrogen, Carlsbad, CA), samples were mixed with TaqMan One-Step RT-PCR Master Mix (Applied Biosystems) and analyzed under the PCR program, consisting of reverse transcription at $48^{\circ} \mathrm{C}$ for 30 minutes, Taq polymerase at $95^{\circ} \mathrm{C}$ for 10 minutes, 40 cycles at $95^{\circ} \mathrm{C}$ for denaturation for 15 seconds, and annealing and extension at $60^{\circ} \mathrm{C}$ for 1 minute. Primer sets and probes (designed using Primer Express 2.0 Software; Applied Biosystems, Weiterstadt, Germany): KDR forward, 5'-CACCACTCAAACGCTGACTA-3'; KDR reverse, 5'-CCAACTGCCAATACCAGTGGAT-3'; KDR probe, 5'-(FAM)TGCCATTCCTCCCCCGCATC(TAMRA)-3'; $V E$-cadherin forward, 5'-GCCATCGATAATTCTGGACGTATT-3'; VE-cadherin reverse, 5'-GAAGATGGTGATGGGATTTC-3'; and VE-cadherin probe, 5'-(FAM)TCACAATAACGAAAAGCTTGGACCG(TAMRA)- $3^{\prime}$. The primers and probe used to amplify and detect glyceraldehyde phosphate dehydrogenase $(G A P D H)$ were from the TaqMan GAPDH Control Reagent kit (Applied Biosystems). The GAPDH was used as an internal standard to normalize cDNA levels. A single aliquot from a total RNA stock extracted from second passage human umbilical vein endothelial cells (HUVECs) kept in frozen aliquots at $-70^{\circ} \mathrm{C}$ was used in each experiment to normalize between experiments. The relative expression of respective genes was calculated by using the comparative threshold cycle $\left(\mathrm{C}_{\mathrm{T}}\right)$ method, as previously described. ${ }^{43}$

\section{RNase protection assay}

The ribonuclease (RNase) protection analysis was done using the RiboQuant Multi-Probe RNase protection assay system (BD BiosciencesPharMingen) as described previously. ${ }^{44}$ Briefly, thalidomide and CC-5013 (kindly donated by Dr Peter Schafer, Celgene) were dissolved in dimethyl sulfoxide (DMSO) (Sigma Chemicals) and stored at $-20^{\circ} \mathrm{C}$ until use. Drugs were diluted in culture medium and used at a range of final concentrations between $10^{-6}$ and $10^{-5} \mathrm{M}$ with less than $0.1 \%$ DMSO. Confluent, second-passage HUVECs were exposed for 18 hours to drugs or to DMSO alone; following extraction of total RNA, $10 \mu \mathrm{g}$ was hybridized to cytokine receptor or angiogenesis antisense probe cocktails (BD BiosciencesPharMingen) exactly as we described previously. ${ }^{44}$ Quantification of mRNA was done from PhosphorImager (Molecular Dynamics, Sunnyvale, CA) analysis of radioactive bands using ImageQuant software (Molecular Dynamics). To control for differences in sample processing, hybridization signals in each sample were divided by the signal for the ribosomal protein mRNA (L32), which by independent analysis was found not to change with exposure to indicated concentrations of drugs or DMSO (data not shown).

\section{Western blotting}

Immunoblotting was performed first with $10 \mu \mathrm{g} / \mathrm{mL}$ rabbit anti-TGFßRI (Santa Cruz Biotechnology, Santa Cruz, CA), then with $20 \mu \mathrm{g} / \mathrm{mL}$ mouse antithrombin receptor (PAR-1; Santa Cruz Biotechnology), and finally with $60 \mu \mathrm{g} / \mathrm{mL}$ rabbit anti-actin (Santa Cruz Biotechnology) to ensure equal loading. Antibody detection was performed by enhanced chemiluminescence (ECL) techniques (Perkin-Elmer, Boston, MA) as we described previously. ${ }^{44}$

\section{Statistical analysis}

The 2-tailed, Student $t$ test was used to compare patient and control CECs. Comparison of groups in remaining experiments was nonpaired analyses using 2-tailed Student $t$ test. Data are presented as means $\pm 1 \mathrm{SD}$. Associations between variables were evaluated by Pearson $r$. Significance level was set at $P$ less than or equal to .05 throughout.

\section{Results}

\section{Patient characteristics}

Demographics and clinical staging of patients are shown in Table 1. The population was predominantly African American female immigrants from the Caribbean. This group may have a predisposition to $\mathrm{MM}$ since, in the general population, $\mathrm{MM}$ is more common in men. At the time of study, all patients were at indicated stages of treatment with thalidomide $(200 \mathrm{mg} / \mathrm{d})$ for symptomatic MM ranging from 0 months (immediately prior to starting treatment) to 30 months. Patients with relapse were not included, since cytotoxic chemotherapy used in relapse treatment modulates cellular functions within bone marrow microenvironment.

\section{Circulating endothelial cells}

CECs were defined as those PBMCs that simultaneously expressed the EC antigens CD105, CD146, and CD34 and were null for the macrophage cell surface marker CD11c (Mac-1). The EC antigens were selected on the basis of their clinical relevance to EC function in disease. ${ }^{45-49}$ Fluorescence-activated cell sorting (FACS) analysis showed that across all patient $(\mathrm{n}=26)$ and control samples $(\mathrm{n}=22)$, more than $98 \%$ of $\mathrm{CD} 105^{+}$cells also expressed membrane CD34 and CD146 antigens (Figure 1A); the frequency of CECs in patients was 6-fold higher than controls $(P<.001)$ (Figure 1B). The distributions of CECs across stage 2 and stage 3 MM were similar, although the highest proportion of CECs was observed in patients with stage 3 disease (Table 1).

The clinical relevance of this finding was indicated by a positive correlation between patients' CEC levels and their serum levels of $\mathrm{M}$ protein $(\mathrm{r}=.62, P=.001)$ and $\beta_{2}$-microglobulin $(\mathrm{r}=.72$, $P<$.001) (Figure 2A-B). This population of patients included both untreated and treated subjects. The correlation between CECs and $\mathrm{M}$ protein in this MM population suggests that CECs and $\mathrm{M}$ protein vary similarly in patients. Therefore, similar to $\mathrm{M}$ protein, which is highly clinically relevant as a measure of tumor load and disease activity before and during treatment, CECs also may be linked to pathogenesis in a way that is relevant throughout the treatment
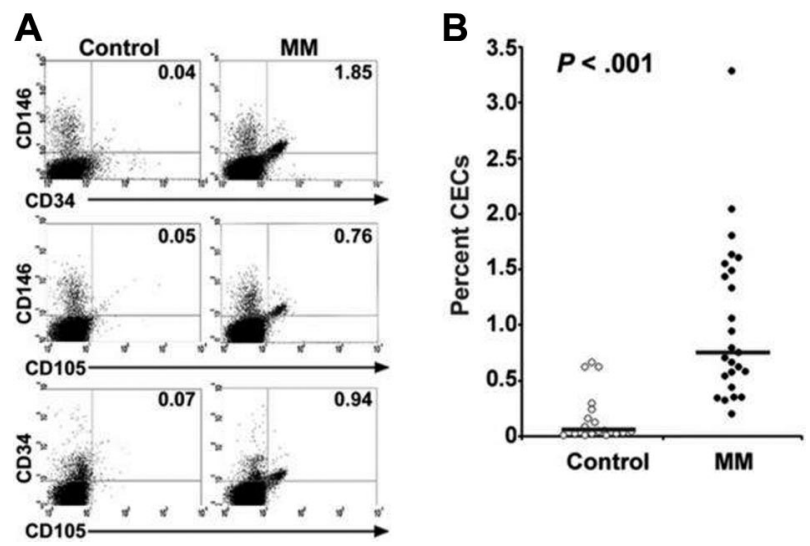

Figure 1. Flow cytometric determination of the frequency of circulating endothelial cells (CECs) in patients with MM compared with healthy control subjects. (A) Peripheral blood mononuclear cells were triple-labeled with anti-CD11bCy, anti-CD105-FITC, and either anti-CD34-PE or anti-CD146-PE; 100000 cells were acquired and analyzed by flow cytometry. Forward-scatter and side-scatter plots were used to identify the mononuclear cell population. Measurements for FITC were carried out using the fluorescence-1 (FL1) detector $(530 / 30 \mathrm{~nm})$, PE was detected using the FL2 detector $(585 / 42 \mathrm{~nm})$, and CyChrome was detected using the FL3 detector $(640 \mathrm{~nm})$. Percent positive cells was compared with the percent of cells positive in staining with PE- or FITC-labeled IgG isotype controls; polyfluorescent beads (DAKO FluoroSpheres; DAKO, Glostrup, Denmark) were used to standardize the cytometer. The percentages of the $\mathrm{CD} 11 \mathrm{~b}^{-}$cell population expressing the indicated double antigens from a representative patient and a control subject are shown in each quadrant. In statistical analyses, CD11b ${ }^{-}$cells expressing CD105 and CD146 were considered CECs. (B) The percentages of CECs in PBMCs from 26 patients with MM (closed circles) and 12 healthy control subjects (open circles) are shown. Horizontal bars indicate the mean values for each group. Results are the mean of at least 2 FACS determinations performed at least 24 hours apart for each person. Group comparisons were made by Student $t$ test, 2-tailed. 
From www.bloodjournal.org by guest on February 1, 2019. For personal use only.

Table 1. Description of the overall population at the time of study

\begin{tabular}{|c|c|c|c|}
\hline Patient characteristics & Stage II, $n=6$ & Stage III, $n=25$ & All patients, ${ }^{\star} n=31$ \\
\hline Median age, y (range) & $49.5(46-73)$ & $62.0(43-84)$ & $61.0(43-84)$ \\
\hline Male:female & $1: 5$ & $5: 20$ & $6: 25$ \\
\hline Median M protein, $\mathrm{mg} / \mathrm{dL}$ (range) $\dagger$ & $800(150-1700)$ & $1200(158-6000)$ & $1200(150-6000)$ \\
\hline Median $\beta_{2}$-microglobulin, mg/L (range) & $1.86(0.96-3.5)$ & $2.47(1.02-12)$ & $2.26(0.96-12)$ \\
\hline \multicolumn{4}{|l|}{ Treatment with thalidomide, $n$} \\
\hline Untreated & 3 & 5 & 8 \\
\hline$\leq 2$ weeks & 0 & 5 & 5 \\
\hline$>2$ weeks and $<4$ months & 0 & 4 & 4 \\
\hline$\geq 4$ months & 3 & 11 & 14 \\
\hline \multicolumn{4}{|l|}{ Median percent CECs } \\
\hline All patients (range) & $0.87(0.58-1.49)$ & $0.64(0.13-2.04)$ & $0.73(0.13-2.04)$ \\
\hline Healthy controls (range) & NA & NA & $0.05(0.1-0.66)$ \\
\hline
\end{tabular}

Staging was done at diagnosis in accordance with Jacobsen et al. ${ }^{42}$

NA indicates not applicable to healthy controls.

"Not all patients underwent all of the studies described in the "Results" section.

†In 2 cases of light chain MM, Bence-Jones protein was considered as M protein.

stages. However, correlation between MM disease parameters such as $\mathrm{M}$ protein and CECs also will be studied longitudinally in patients before and subsequent to treatment to determine the CECs' relationship with MM activity.

\section{Characterization of EPCs}

A distinguishing feature of EPCs is their late formation of colonies (after day 14 in culture) compared to more differentiated ECs, which form colonies after only 7 to 10 days in culture. ${ }^{21}$ Similar to mature ECs, EPCs forming colonies subsequently outgrow the colony confines into confluent monolayers. In addition, in vitro EPCs grow into 3-dimensional cultures forming capillary-like tubes and networks. Since CECs were increased in patients, it was essential to characterize circulating EPCs within this population in order to determine their role in neovasculogenesis associated with MM.

We assessed the relative frequency of EPCs in patients versus controls by measuring the number of CFUs generated from PBMCs grown on laminin-coated plates. In this assay, as shown in Figure $3 \mathrm{~A}$, distinct colonies of elongated ECs radiating from a cluster of round cells were observed 14 to 18 days after plating (i); colony size and numbers gradually decreased (ii), followed by endothelial confluency by 20 to 28 days (iii); within a time frame similar to that seen with laminin cultures, colony formation was observed on cells grown on matrigel (iv); subsequent tube formation (v) and a dense capillary-like network formation (vi) also were observed. These results establish the presence of angioblastlike EPCs circulating in peripheral blood of patients with MM. In contrast to our findings in 19 patients in whom colony growth was studied, when PBMCs from 7 healthy subjects were cultured under conditions identical to those producing data shown in Figure 3, late colony formation was observed in only 1 subject in whom mean \pm SD CFU count on day 14 on laminin wells was $2.5 \pm 0.6$ compared to the colony counts from patients shown in Figure 3B. Quantitation of colony numbers showed that parallel to the frequency of CECs in blood, colony numbers were closely related to $\mathrm{MM}$ activity indicated by $\mathrm{M}$ protein levels. As demonstrated in Figure 3B, mean colony numbers were high in the 3 untreated patients and also in the 8 patients in whom $\mathrm{M}$ protein levels decreased only by $25 \%$ or less after 3 to 12 months of thalidomide treatment. In contrast, colony numbers were lower in patients $(n=8)$ in whom a clinically better response to thalidomide was achieved, indicated by a more than $25 \%$ decrease in M protein. Others have found that ECs from the bone marrow of patients with MM show intense network formation on matrigel, correlating with the degree of bone marrow angiogenesis. ${ }^{17}$ We thus studied EPC growth on matrigel in order to determine whether peripheral blood ECs similarly would display network formation, which would be indicative of an increase in tumor angiogenesis in vivo. In patients who had received no thalidomide or up to 2 weeks of thalidomide treatment $(n=12)$, network formation covered $60 \% \pm 2.5 \%$ of the surface area of the wells, whereas in other patients who had received thalidomide for 3 months $(n=4)$, network formation covered only $20 \% \pm 4.2 \%$ of the wells (Figure 3C, representative data for 1 of 12 patients). Of 12 patients who had not received thalidomide, 3 were studied at 3 months and 6 months after thalidomide treatment for capillary network formation. As shown in Figure 3D, thalidomide induced a progressive decrease of in vitro vascularization in matrigel in the patients. These results suggest that suppression of circulating EPCs could be a key mechanism responsible for effectiveness of thalidomide in MM.

As shown in Figure 4, the endothelial origin of CFUs and confluent EPCs was confirmed by immunocytochemistry performed at the indicated time points (shown on the left) after plating the cells on laminin. The EC colonies observed on day 14 were
Figure 2. Association between clinical parameters of MM and percentage of CECs. (A) The percentage of CECs was positively correlated with patients' levels of serum $M$ protein and $(B) \beta_{2}$-microglobulin $(n=26)$. CEC frequency was determined by FACS analysis. Association between variables was evaluated by Pearson $r$.
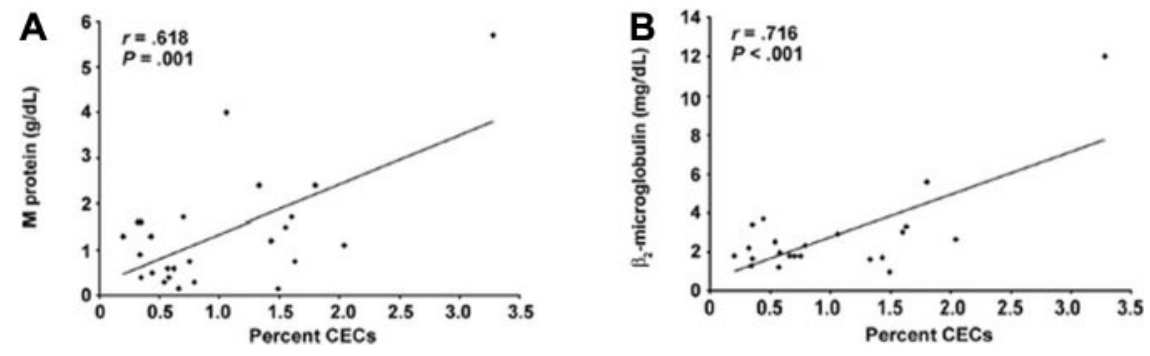

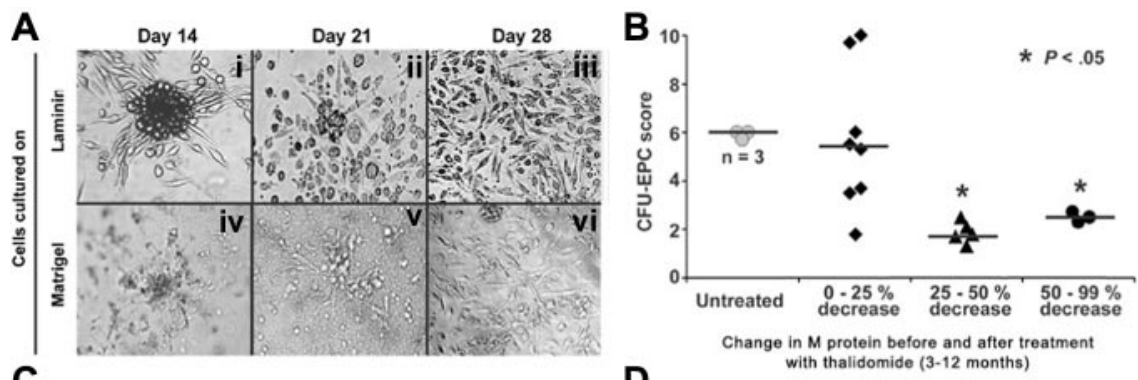

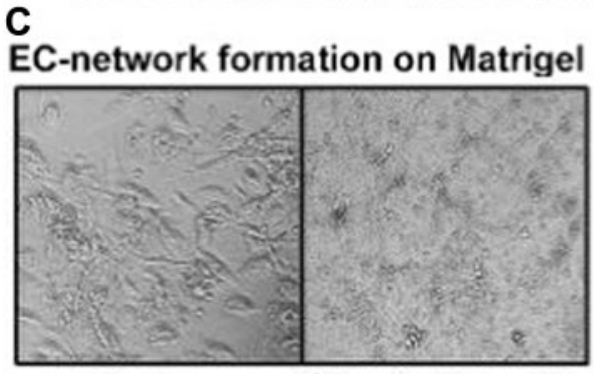

Before After thalidomide
D

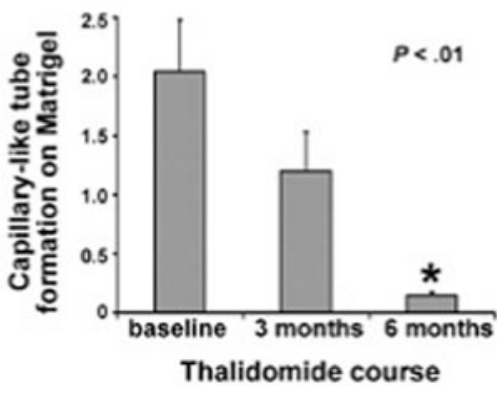

Figure 3. In vitro studies of endothelial progenitor cell (EPC) colony formation, growth, and capillary-like network formation before and after thalidomide. (A) Peripheral blood mononuclear cells were cultured in laminin-coated 6-well plates (i-iii) or in matrigel-coated 48-well plates (iv-vi). Colonies were quantitated 14 days after plating. Phase contrast micrographs of cultures from an MM patient were examined at the indicated time points: (i) an EPC colony characterized by a central cluster of rounded cells surrounded by radiating, thin, flat, elongated cells; (ii) smaller colonies at the indicated time point and a reciprocal increase in EC outgrowth; (iii) confluent expansion of endothelial cells; (iv) colony growth and tube formation on matrigel; (v) progression of tube formation; and (vi) EC network formation. Original magnification $\times 200$. (B) Colony formation on laminin from patients with $\mathrm{MM}$, stratified by change in M protein levels after treatment with thalidomide. $P$ was determined by nonpaired analysis using Student $t$ test. (C) Comparison of capillary-like network formation by EPCs from a patient before and after 3 months of treatment with thalidomide. The patient's serum M protein levels had decreased by $75 \%$ compared to pretreatment (not shown). PBMCs were grown on matrigel. Original magnification $\times 200$. (D) Time course of thalidomide's effect on EC network formation. Bars represent mean \pm SD of 3 patients' capillary-like tube formation scores which were determined sequentially at the indicated times during treatment with thalidomide. Tube formation score was assigned by phase contrast microscopy as described in "Patients, materials, and methods." Mean of triplicate wells from each patient at indicated times was assigned as their score.

strongly positive for KDR and expressed lower levels of VEcadherin (Figure 4A); expression of VE-cadherin and VWF was abundant in smaller colonies and in outgrowing EPCs on day 21 (Figure 4B), outgrowing ECs were similarly characterized by their intense staining for KDR and co-expression of CD133 (Figure 4C). This is particularly important since CD133 expression is gradually lost during EC differentiation and is absent from mature ECs. ${ }^{31,50}$ Outgrowing EPCs at day 28 also expressed VWF; expression of VE-cadherin was more concentrated at the poles in these cells (Figure 4D-F), consistent with the function of this antigen as an inter-EC adhesion molecule. CFUs also expressed CD105, CD146, CD31, and CD34 at lesser intensity and did not express CD38, a membrane protein present on plasma cells (data not shown).

Since VEGF in bone marrow microenvironment drives EC proliferation and angiogenesis largely by signaling through KDR, its gene expression was studied further by comparing mRNA levels of patients and controls. In parallel to its strong expression on the EPC membrane, KDR mRNA levels in patients with MM were higher than in controls, while expression of VE-cadherin was not increased (Figure 5A). As shown in Figure 5B, while KDR mRNA levels showed variation, it was significantly elevated in patients $(\mathrm{n}=21)$ compared to controls $(\mathrm{n}=6)(P=.005)$, and the highest levels occurred in patients with concurrently high $\mathrm{M}$ protein levels (Figure 5C). The clinical significance of elevation in KDR mRNA in peripheral blood mononuclear cells from patients with MM was further underscored by demonstration of its positive correlation with serum $\mathrm{M}$ protein in these patients $(r=.595, P=.003)$ (Figure 5C). Recent data indicate that MM cell lines and patient cells express the KDR gene and protein. ${ }^{51}$ Thus, MM cells within the PBMCs in circulation could be the source of $K D R$ mRNA obtained from patients. However, flow cytometry and immunocyto- chemistry data indicated the absence of cells that coexpressed KDR and CD38 in patients (data not shown), indicating that plasma cells could not be the major source of the KDR mRNA in the population we studied.

\section{Effects of thalidomide and the IMiD CC-5013 on EC gene expression}

Since CECs and EC colony and capillary-like network formation were significantly decreased in patients receiving thalidomide, we studied the effects of this class of agents on expression of a panel of key genes that regulate angiogenesis. For this purpose, effects of thalidomide and CC-5013 on HUVECs were studied by quantitative mRNA expression analysis using RNase protection assays (Figure 6A-B). Compared to controls treated with DMSO, exposure of HUVECs overnight to thalidomide or CC-5013 at a $10^{-6}$ molar concentration resulted in a significant reduction in mRNA levels for TGF $\beta R I, I L-1 R I, T N F-\alpha$ receptor $p 75$, and $g p 130$, a receptor for IL-6 family that mediates tumor growth in MM (Figure 6C). Receptors that were inhibited to a lesser but statistically significant extent included TR, Tie 2 , and TNF- $\alpha$ receptor $p 55$. These findings are consistent with previously described inhibitory effects of thalidomide and CC-5013 on TNF- $\alpha$ receptors, ${ }^{52} I L$ $1 R I,{ }^{53}$ Tie $2,{ }^{54}$ and also on $g p 130 .{ }^{55}$ The inhibitory effects of thalidomide or CC-5013 on TGFßRI gene expression have not been described previously, to our knowledge. This finding is significant because of up-regulation of TGF $\beta R I$ in bone marrow $\mathrm{EC}$ in patients with $\mathrm{MM}^{17}$ and suggests that this ser/thr kinase receptor or its downstream intracellular mediators SMAD proteins may be one mechanism that regulates angiogenesis in MM. Similarly, inhibitory effects of thalidomide or CC-5013 on TR gene 

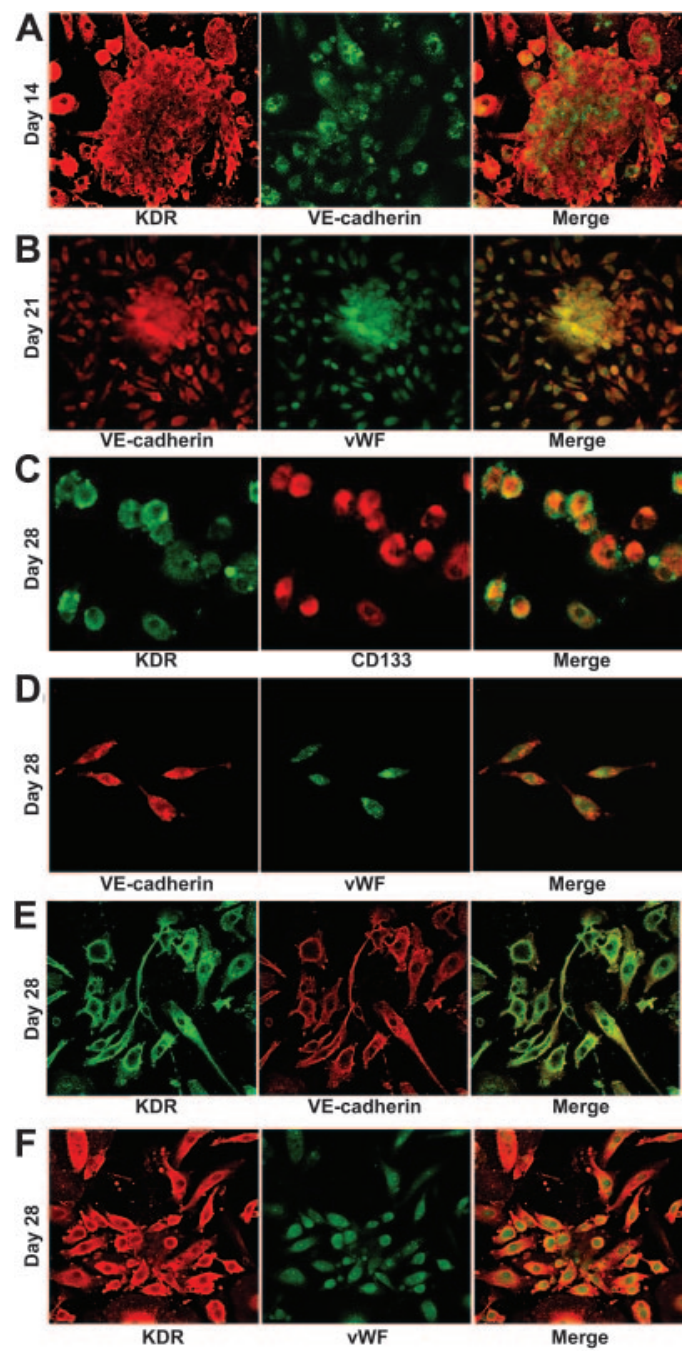

Figure 4. Characterization of EPCs expressing KDR, vascular endothelial cadherin (VE-cadherin), von Willebrand factor (VWF), and CD-133. Colonies and outgrowing cells were characterized by their high expression of KDR compared with other antigens. A representative panel of studies from 1 patient is shown. (A) A large colony manifests predominant and intense expression of membrane KDR (red) and less intense expression of VE-cadherin staining by a smaller percentage of cells (green). (B) Colonies grew smaller by day 21, expressed VWF (green), and showed increased VE-cadherin expression (red). (C) Outgrowing EPCs expressed CD133 (red) and KDR (green). (D) Compared with VWF (green), VE-cadherin (red) was also localized to the poles of outgrowing EPCs. (E) Outgrowing EPCs had consistently intense staining for KDR (red), and all cells also expressed VE-cadherin (green) and (F) VWF (green). Cultures were maintained on 96-well laminin-coated plates in M199 medium containing $50 \mu \mathrm{g} / \mathrm{mL}$ heparin, $50 \mu \mathrm{g} / \mathrm{mL}$ endothelial cell growth medium, and $20 \%$ fetal calf serum at standard culture conditions and were fixed for immunostaining at the time points indicated on the left. Images were generated at the projections of the $z$-stacks at $1024 \times 1024$ pixels.

expression is also of interest because inhibition of $T R$ gene expression could interfere with antiangiogenic actions of thrombin. When these actions of thrombin are considered, such as potentiation of VEGF-induced EC proliferation, up-regulation of VEGF receptors KDR and flt-1, the flt-1 ligand $\alpha_{\mathrm{V}} \beta_{3}$ integrin, VEGF secretion, and expression of matrix metalloprotease-9 (MMP9), ${ }^{56}$ it is possible that signaling via TR could contribute to angiogenesis in MM. Lastly, as shown in Figure 6D, which represents 1 of 2 independent experiments that yielded similar results, Western blot analysis showed that the effects of thalidomide and CC-5013 on mRNA levels of TGF $\beta R I$ and TR genes in HUVECs are paralleled by a simultaneous decrease in their respective protein levels. Furthermore, a reducing effect of thalidomide and CC-5013 on expression of the 2 proteins was also observed in primary EPCs outgrown from PBMCs from 2 untreated patients, of which 1 is shown in Figure 6D.

\section{Discussion}

We show that in MM, (1) CECs are increased in blood and covary with 2 major clinical indicators of disease activity, for example, levels of $\mathrm{M}$ protein and $\beta_{2}$-microglobulin; (2) among CECs are EPCs with angioblastic and neovascularization capabilities; and (3) clinical response to thalidomide, defined as reduction in levels of $M$ protein and $\beta_{2}$-microglobulin, is associated with a parallel decline in CECs and EPCs. We also suggest that EC pathology in MM may involve the TGF $\beta$ RI pathway, since this gene was targeted by thalidomide and CC-5013 in HUVECs and patient EPCs.

The covariation of CECs and EPCs with M protein and $\beta_{2}$-microglobulin supports the clinical relevance of these vascular markers in reflecting tumor mass and prognosis in MM. The current findings support the concept that by virtue of their mobilization, EPCs represent a surrogate indicator of angiogenesis and of response to antitumor strategies. ${ }^{20,38}$ On the basis of these results, it is possible to hypothesize that, similar to $M$ protein and $\beta_{2^{-}}$ microglobulin, CECs and EPCs reflect disease progression or regression and are relevant parameters in both untreated and treated patients. This thesis will be explored in a larger longitudinal study.
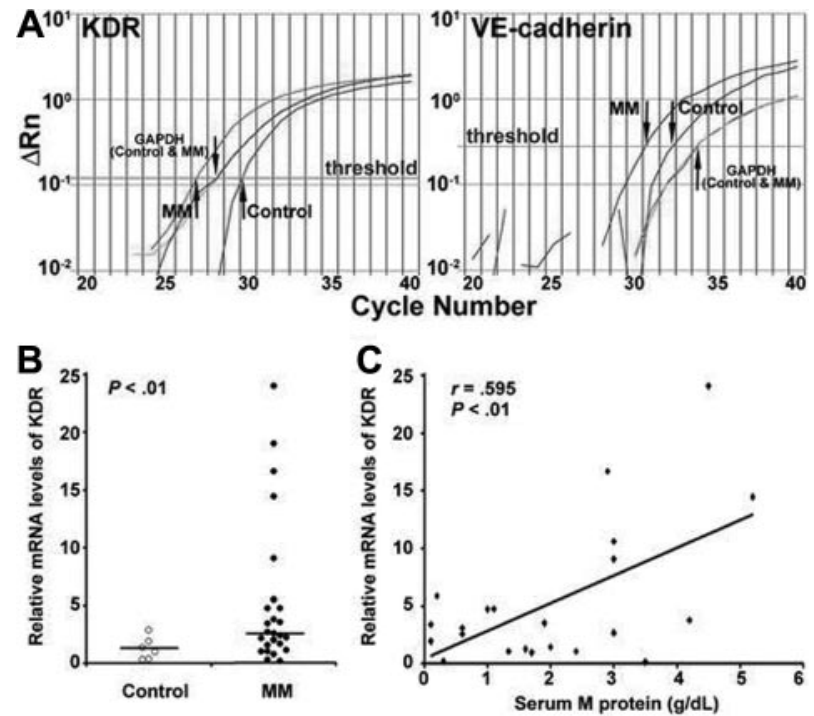

Figure 5. Quantitative measurement of $K D R$ and VE-cadherin gene expression in MM using real-time RT-PCR. The CDNA of PBMCs from patients with MM or controls was used to amplify KDR, VE-cadherin, and GAPDH in real-time PCR reactions. An optimized threshold line was determined in the range of linear amplification to compare cycle number in different samples. The expression of $K D R$ and VE-CADHERIN was calculated by the comparative threshold cycle method. Reproducibility of this method was confirmed by repetition of each experiment once using frozen aliquots of the same mRNA extracted within 6 hours of collection of PBMCs from subjects and stored at $-20^{\circ} \mathrm{C}$ for 4 weeks. (A) Representative amplification plots for $K D R$ (left) or VE-cadherin (right) expression in PBMCs from an MM patient and a control subject; GAPDH amplification was used as an internal control. While GAPDH levels for both MM and control are comparable, indicating the same amount of cDNA used in each reaction, expression of $K D R\left(\mathrm{C}_{\mathrm{T}}=30.2\right.$ for control, $\mathrm{C}_{\mathrm{T}}=26.3$ for $\left.\mathrm{MM}\right)$ and VE-cadherin $\left(\mathrm{C}_{\mathrm{T}}=32.9\right.$ for control, $\mathrm{C}_{\mathrm{T}}=31.1$ for $\mathrm{MM}$ ) were different. Arrows indicate the $\mathrm{C}_{\mathrm{T}}$ values. (B) Relative mRNA expression of $K D R$ is shown for patients (filled circles) and controls (open circles). (C) KDR mRNA level is positively correlated with concurrently determined serum $M$ protein levels. The association between KDR mRNA and M protein was evaluated by Pearson $r$. 

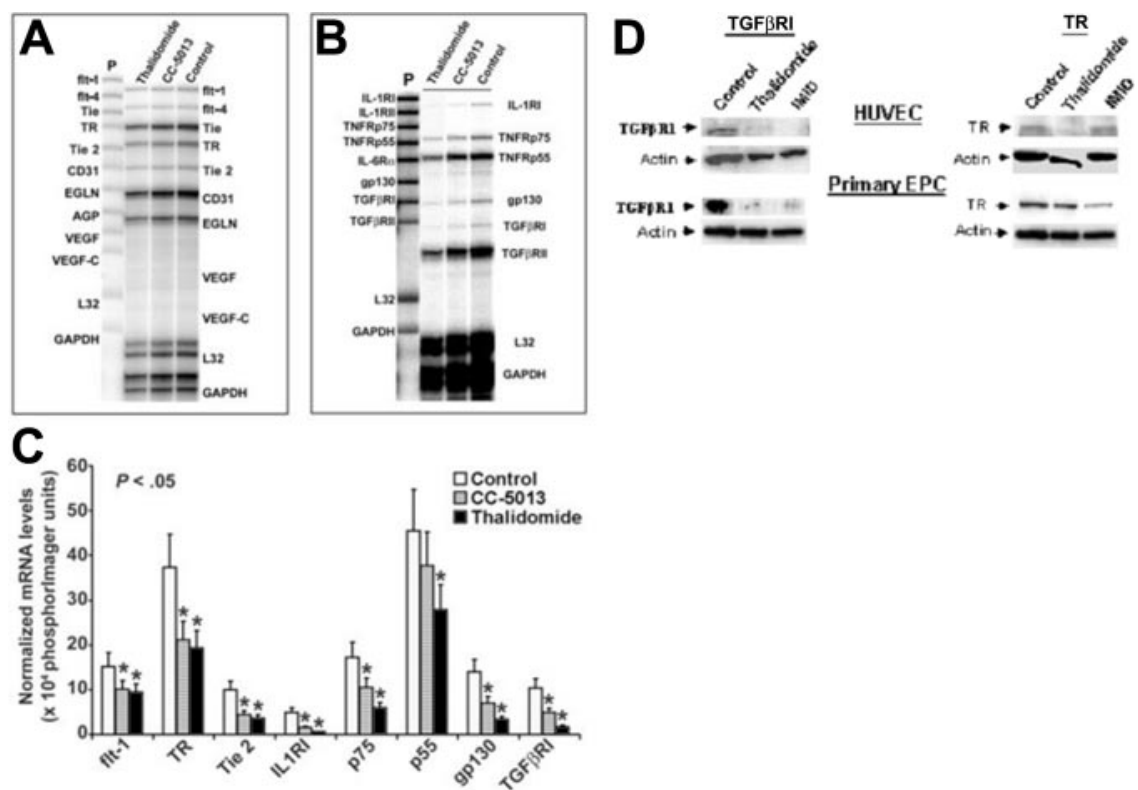

Figure 6. The effect of thalidomide and CC-5013 on angiogenic and cytokine receptor expression in HUVECs. (A-C) RNase protection analyses of HUVEC mRNA are shown. Total RNA was extracted after 18 hours of exposure to $10^{-6} \mathrm{M}$ thalidomide, CC-5013, or control medium. Ten micrograms total RNA was hybridized to either (A) an angiogenesis probe set containing templates for angiopoietin (ANG), CD31 (platelet endothelial cell adhesion molecule-1 [PECAMI), endoglin, VEGF receptor-1, fms-like tyrosine kinase (flt-1), Ang receptors (TIE-1 and TIE-2), VEGF, and VEGF-C; or (B) a receptor probe set containing the templates for interleukin-1 receptor I (IL-1RI), IL-1RII, tumor necrosis factor- $\alpha$ receptor p55 (TNFRp55), TNFRp75, IL-6R $\alpha$, gp130, TGF $\beta R I$, and TGF $\beta R / l$ genes. (C) Three independent sets of experiments yielded similar data. The graph represents the quantification summary for each probe. Gene signals were normalized to that from L32 (mRNA for a ribosomal protein subunit). White bars represent gene expression in untreated HUVECs, while gray and black bars represent gene expression in HUVECs treated with CC-5013 and thalidomide, respectively. Comparisons between relative Phosphorlmager units for control and experimental samples for each gene were made by 2-tailed Student $t$ test. ${ }^{\star} P<.05$. (D) Western blot analysis of HUVECs and primary EPC whole-cell lysates. HUVECs and primary EPCs outgrown from PBMCs isolated from 2 untreated patients were cultured to confluence in gelatin- and laminin-coated 6-well plates, respectively. Data from 1 of 2 experiments with similar results are shown. Lysates from control and treated ECs are shown. Following electrophoresis and blotting, the membranes were developed by means of antibodies specific for either rabbit anti-TGF $\beta$ RI or mouse anti-TR, followed by chemiluminescence and autoradiography. The membranes were stripped and developed similarly with rabbit anti-actin to control for equal loading.

Although we did not directly measure the proliferative rate of EPCs, we observed a significant increase in the level of colony growth in EPCs from patients compared to control subjects. We found that EPCs were characterized by strong expression of KDR compared to other EC antigens, including VE-cadherin and VWF, which EPCs uniformly expressed at lesser levels. Thus, EPCs in MM bear similarity to embryonic angioblasts, which express high levels of KDR. The receptor KDR binds VEGF with lesser affinity than Flt1, but KDR mediates most actions of VEGF on angioblasts, including survival, migration, and tube formation. ${ }^{34}$ Recent data show that EPCs from cord blood express high levels of KDR, resulting in their increased sensitivity to the angiogenic effects of VEGF. ${ }^{57}$ Similarly, in MM, increased KDR expression by EPCs suggests the possibility of increased sensitivity of these cells to VEGF; such sensitivity might account for our finding of growth of EPCs from patients with MM but not from healthy controls, since the EPCs could not survive below a critical concentration of VEGF (which was not added exogenously to cultures). Whether $K D R$ expression in MM EPCs is an autologous response to endothelial VEGF production is not known. Up-regulation of endothelial KDR gene expression is caused by hypoxia ${ }^{58,59}$ and transformation with human herpes virus $8,{ }^{60}$ but the role of these factors in MM pathogenesis is not known. High KDR gene and protein expression suggests that this receptor plays a role in the expansion or mobilization of EPCs in MM. The relationship between levels of $K D R$ mRNA and $\mathrm{M}$ protein supports this notion and further indicates that EPCs in MM have either maintained or transformed to their phenotypic and functional prenatal status. We are aware, however, that future studies of EPCs in MM should use stringent cell separation techniques since KDR is also expressed by MM cells and cell lines. ${ }^{51}$
The present results suggest that a suppressive effect of thalidomide on circulating EPCs might be the key mechanism by which this drug affects angiogenesis in MM. The results also indicate that patients in whom thalidomide is ineffective can be identified by high colony growth and high $\mathrm{M}$ protein levels. These patients also have the highest $K D R$ mRNA levels, indicating that the effects of thalidomide are largely VEGF dependent. Whether thalidomide induces apoptosis or has cytotoxic effects on CECs and EPCs in patients with $\mathrm{MM}$ is yet to be determined. In addition, both thalidomide and, especially, CC-5013 have potent immunomodulatory effects, the most significant of which are suppression of TNF- $\alpha$ and activation of $\mathrm{T}$ cells at different stages of this process. ${ }^{61-63}$ Since evidence indicates that inhibition of angiogenesis requires intact $\mathrm{CD}^{+}{ }^{+}$and $\mathrm{CD} 8^{+} \mathrm{T}$-cell function, ${ }^{64}$ the degree to which immune restoration is a mechanism by which these drugs modulate CEC numbers, EPC colony formation, and/or tumor neovascularization remains to be elucidated. Since thalidomide effects are mediated by its metabolites, a precise comparison between effects of thalidomide and CC-5013 on EPCs is difficult to assess accurately in vitro; this will be achieved in future in vivo studies in patients with MM on treatment.

Increased levels of EPCs and their corresponding gene and protein expression profiles in MM might result from processes such as expansion of genetically altered EPCs harboring chromosomal mutations or clonal growth in MM via mechanisms similar to those operating in MM cells. ${ }^{65}$ For example, a population of ECs in chronic myeloid leukemia was shown to harbor the breakpoint cluster region/ABL $(B C R / A B L)$ fusion gene. ${ }^{66}$ If such EPCs are found in $\mathrm{MM}$, this may suggest that that a clonal hemangioblastic progenitor could be part of the neoplastic process, which may constitute the mechanism, in addition to those already suggested, 
by which single and tandem bone marrow transplantation in patients with MM is successful. ${ }^{67,68}$

In the present study, we found that thalidomide and CC-5013 regulated the expression of ser/thr kinase TGFßRI, which in cooperation with HIF-1 $\alpha$ stimulates VEGF gene expression induced by hypoxia, which promotes angiogenesis. ${ }^{44,69}$ Gene expression of TGF $\beta R I$ and $H I F-1 \alpha$ were recently shown to be elevated in bone marrow ECs in patients with MM. ${ }^{17,54}$ Furthermore, the regulatory effects of TGF $\beta$ RI and HIF- $1 \alpha$ on angiogenesis, bone development, and progression of skeletal metastases ${ }^{39,70-73}$ suggest that both genes also may affect MM cells' interactions with their microenvironment at several levels.

A second target of thalidomide and CC-5013 treatment of HUVECs was the $T R$, a member of the G-protein-coupled protease-activated receptors (PAR) expressed on MM cell lines and normal plasma cells but not on B cells. ${ }^{74}$ Activation of the TR by thrombin induces cleavage and shedding of syndecan- $1,{ }^{75}$ which indicates poor prognosis in MM. ${ }^{76}$ Recent data indicate that in human coronary artery ECs damaged by exposure to doxorubicin, thalidomide increases TR expression, suggesting that this is a mechanism by which thalidomide's known side effect of increased thrombosis could occur. ${ }^{77}$ The difference between our results probably lies in differences in EC activation at the time of the studies. (Our studies used low-passage HUVECs, which were found by immunofluorescence to be E-selectin negative [data not shown], indicating that the cells were not activated.)

We conclude that increased EPCs in circulation represent an extension of the tumor microenvironment in MM. EPCs in MM express high levels of $K D R$ and resemble embryonic angioblasts. The ready accessibility of EPCs will help further the study of the MM-specific aspects of EC biology, the evaluation of disease activity, and the monitoring clinical response, thereby further elucidating the pathways and mechanisms that affect MM angiogenesis and progression.

\section{Acknowledgments}

We thank Amal Ibrahim, Division of Hematology, for excellent technical assistance; and Mary Mondragon-Escorpizo, Transplant Immunology, and William Oxberry, Department of Pathology, at SUNY Downstate for their excellent and dedicated technical support. Doruk Gölcü, Bilkent University, performed preliminary experiments for KDR mRNA studies. Dr Jin Zhao participated in preliminary flow cytometry experiments.

\section{References}

1. Anderson KC. Moving disease biology from the lab to the clinic. Cancer. 2003;97:796-801.

2. Vacca A, Ribatti D, Roncali L, et al. Bone marrow angiogenesis and progression in multiple myeloma. Br J Haematol. 1994;87:503-508.

3. Rajkumar SV, Mesa RA, Fonseca R, et al. Bone marrow angiogenesis in 400 patients with monoclonal gammopathy of undetermined significance, multiple myeloma, and primary amyloidosis. Clin Cancer Res. 2002;8:2210-2216.

4. Pruneri G, Ponzoni M, Ferreri AJ, et al. Microvessel density, a surrogate marker of angiogenesis, is significantly related to survival in multiple myeloma patients. Br J Haematol. 2002;118:817820.

5. Sezer O, Niemoller K, Kaufmann O, et al. Decrease of bone marrow angiogenesis in myeloma patients achieving a remission after chemotherapy. Eur J Haematol. 2001;66:238-244.

6. Rajkumar SV, Fonseca R, Witzig TE, Gertz MA, Greipp PR. Bone marrow angiogenesis in patients achieving complete response after stem cell transplantation for multiple myeloma. Leukemia. 1999;13:469-472.

7. Bellamy WT, Richter L, Frutiger Y, Grogan TM Expression of vascular endothelial growth factor and its receptors in hematopoietic malignancies. Cancer Res. 1999;59:728-733.

8. Vacca A, Ribatti D, Presta M, et al. Bone marrow neovascularization, plasma cell angiogenic potential, and matrix metalloproteinase-2 secretion parallel progression of human multiple myeloma. Blood. 1999;93:3064-3073.

9. Anderson KC. Moving disease biology from the laboratory to the clinic. Semin Oncol. 2002;29:1720.

10. Dankbar B, Padro T, Leo R, et al. Vascular endothelial growth factor and interleukin-6 in paracrine tumor-stromal cell interactions in multiple myeloma. Blood. 2000;95:2630-2636.

11. D'Amato RJ, Loughnan MS, Flynn E, Folkman J. Thalidomide is an inhibitor of angiogenesis. Proc Natl Acad Sci U S A. 1994;91:4082-4085.

12. Singhal S, Mehta J, Desikan R, et al. Antitumor activity of thalidomide in refractory multiple myeloma. N Engl J Med. 1999;341:1565-1571.
13. Kumar S, Witzig TE, Dispenzieri A, et al. Effect of thalidomide therapy on bone marrow angiogenesis in multiple myeloma. Leukemia. 2004;18: 624-627.

14. Jaslow R, Kaplan G, Lyons L, Michaeli J, Coleman M. Thalidomide in multiple myelomafrom the clinic to the laboratory. Cancer Invest. 2002;20:1051-1058.

15. Richardson PG, Schlossman RL, Weller E, et al. Immunomodulatory drug CC-5013 overcomes drug resistance and is well tolerated in patients with relapsed multiple myeloma. Blood. 2002; 100:3063-3067.

16. Lentzsch S, LeBlanc R, Podar K, et al. Immunomodulatory analogs of thalidomide inhibit growth of Hs Sultan cells and angiogenesis in vivo. Leukemia. 2003;17:41-44.

17. Vacca A, Ria R, Semeraro F, et al. Endothelial cells in the bone marrow of patients with multiple myeloma. Blood. 2003;102:3340-3348.

18. Folkman J. Angiogenesis-dependent diseases. Semin Oncol. 2001;28:536-542.

19. Carmeliet $P$, Jain RK. Angiogenesis in cancer and other diseases. Nature. 2000;407:249-257.

20. Rafii S. Circulating endothelial precursors: mystery, reality, and promise. J Clin Invest. 2000;105: 17-19.

21. Rafii S, Lyden D. Therapeutic stem and progenitor cell transplantation for organ vascularization and regeneration. Nat Med. 2003;9:702-712.

22. Lyden D, Hattori K, Dias S, et al. Impaired recruitment of bone-marrow-derived endothelial and hematopoietic precursor cells blocks tumor angiogenesis and growth. Nat Med. 2001;7:11941201.

23. de Bont ES, Guikema JE, Scherpen F, et al. Mobilized human $\mathrm{CD} 34^{+}$hematopoietic stem cells enhance tumor growth in a nonobese diabetic/ severe combined immunodeficient mouse model of human non-Hodgkin's lymphoma. Cancer Res. 2001;61:7654-7659.

24. Peichev M, Naiyer AJ, Pereira D, et al. Expression of VEGFR-2 and AC133 by circulating human CD $34^{+}$cells identifies a population of functional endothelial precursors. Blood. 2000;95: 952-958
25. Reyes M, Dudek A, Jahagirdar B, Koodie L, Marker PH, Verfaillie CM. Origin of endothelial progenitors in human postnatal bone marrow. J Clin Invest. 2002;109:337-346.

26. Lin Y, Weisdorf DJ, Solovey A, Hebbel RP. Origins of circulating endothelial cells and endothelial outgrowth from blood. J Clin Invest. 2000;105: 71-77.

27. Pelosi E, Valtieri M, Coppola S, et al. Identification of the hemangioblast in postnatal life. Blood. 2002;100:3203-3208.

28. Yamashita J, Itoh $\mathrm{H}$, Hirashima M, et al. Flk1positive cells derived from embryonic stem cells serve as vascular progenitors. Nature. 2000;408: 92-96.

29. Asahara $\mathrm{T}$, Murohara $\mathrm{T}$, Sullivan $\mathrm{A}$, et al. Isolation of putative progenitor endothelial cells for angiogenesis. Science. 1997;275:964-967.

30. Asahara T, Masuda H, Takahashi T, et al. Bone marrow origin of endothelial progenitor cells responsible for postnatal vasculogenesis in physiological and pathological neovascularization. Circ Res. 1999;85:221-228.

31. Gehling UM, Ergun S, Schumacher U, et al. In vitro differentiation of endothelial cells from AC133-positive progenitor cells. Blood. 2000;95: 3106-3112.

32. Bagley RG, Walter-Yohrling J, Cao X, et al. Endothelial precursor cells as a model of tumor endothelium: characterization and comparison with mature endothelial cells. Cancer Res. 2003;63: 5866-5873.

33. Coffin JD, Harrison J, Schwartz S, Heimark R. Angioblast differentiation and morphogenesis of the vascular endothelium in the mouse embryo. Dev Biol. 1991;148:51-62.

34. Caprioli A, Minko K, Drevon C, Eichmann A, Dieterlen-Lievre F, Jaffredo T. Hemangioblast commitment in the avian allantois: cellular and molecular aspects. Dev Biol. 2001;238:64-78.

35. Shi Q, Rafii S, Wu MH, et al. Evidence for circulating bone marrow-derived endothelial cells. Blood. 1998;92:362-367.

36. Beerepoot LV, Mehra N, Vermaat JS, Zonnenberg BA, Gebbink MF, Voest EE. Increased levels of viable circulating endothelial cells are an indicator 
of progressive disease in cancer patients. Ann Oncol. 2004;15:139-145.

37. Hill JM, Zalos G, Halcox JP, et al. Circulating en dothelial progenitor cells, vascular function, and cardiovascular risk. N Engl J Med. 2003;348:593600.

38. Schuch G, Heymach JV, Nomi M, et al. Endostatin inhibits the vascular endothelial growth factorinduced mobilization of endothelial progenitor cells. Cancer Res. 2003;63:8345-8350.

39. Dickson MC, Martin JS, Cousins FM, Kulkarni AB, Karlsson S, Akhurst RJ. Defective haematopoiesis and vasculogenesis in transforming growth factor-beta 1 knock out mice. Development. 1995; 121:1845-1854.

40. Roberts AB, Wakefield LM. The two faces of transforming growth factor beta in carcinogenesis. Proc Natl Acad Sci U S A. 2003;100:8621. 8623.

41. De Vos J, Couderc G, Tarte K, et al. Identifying intercellular signaling genes expressed in malignant plasma cells by using complementary DNA arrays. Blood. 2001;98:771-780.

42. Jacobson JL, Hussein MA, Barlogie B, Durie BG, Crowley JJ. A new staging system for multiple myeloma patients based on the Southwest Oncology Group (SWOG) experience. Br J Haematol. 2003;122:441-450.

43. Liu W, Saint DA. A new quantitative method of real time reverse transcription polymerase chain reaction assay based on simulation of polymerase chain reaction kinetics. Anal Biochem. 2002 302:52-59.

44. Zhang H, Akman HO, Smith ELP, Zhao J, Murphy-Ullrich JE, Batuman OA. Cellular response to hypoxia involves signaling via Smad proteins. Blood. 2003:101:2253-2260.

45. Cheifetz S, Bellon T, Cales C, et al. Endoglin is a component of the transforming growth factor-beta receptor system in human endothelial cells. J Biol Chem. 1992;267:19027-19030.

46. Azuma H. Genetic and molecular pathogenesis of hereditary hemorrhagic telangiectasia. J Med Invest. 2000;47:81-90

47. Solovey AN, Gui L, Chang L, Enenstein J, Browne PV, Hebbel RP. Identification and functional assessment of endothelial P1H12. J Lab Clin Med. 2001;138:322-331.

48. Solovey A, Lin Y, Browne P, Choong S, Wayner E, Hebbel RP. Circulating activated endothelial cells in sickle cell anemia. N Engl J Med. 1997;337: 1584-1590

49. Fina L, Molgaard HV, Robertson D, et al. Expression of the CD34 gene in vascular endothelial cells. Blood. 1990;75:2417-2426.
50. Peichev M, Naiyer AJ, Pereira D, et al. Expression of VEGFR-2 and AC133 by circulating human CD34(+) cells identifies a population of functional endothelial precursors. Blood. 2000;95 952-958.

51. Kumar S, Witzig TE, Timm M, et al. Expression of VEGF and its receptors by myeloma cells. Leukemia. 2003;17:2025-2031.

52. Marriott JB, Clarke IA, Dredge K, Muller G, Stirling D, Dalgleish AG. Thalidomide and its analogues have distinct and opposing effects on TNF-alpha and TNFR2 during co-stimulation of both CD4(+) and CD8(+) T cells. Clin Exp Immunol. 2002;130:75-84

53. Jin SH, Kim TI, Han DS, Shin SK, Kim WH. Thalidomide suppresses the interleukin 1beta-induced NFkappaB signaling pathway in colon cancer cells. Ann N Y Acad Sci. 2002;973:414-418

54. Giuliani N, Colla S, Lazzaretti M, et al. Proangiogenic properties of human myeloma cells: production of angiopoietin-1 and its potential relationship to myeloma-induced angiogenesis. Blood. 2003;102:638-645.

55. Hideshima T, Chauhan D, Richardson $P$, et al. NF-kappa B as a therapeutic target in multiple myeloma. J Biol Chem. 2002;277:16639-16647.

56. Tsopanoglou NE, Maragoudakis ME. Role of thrombin in angiogenesis and tumor progression. Semin Thromb Hemost. 2004;30:63-69.

57. Bompais $\mathrm{H}$, Chagraoui J, Canron X, et al. Human endothelial cells derived from circulating progenitors display specific functional properties compared with mature vessel wall endothelial cells. Blood. 2004:103:2577-2584.

58. Brogi E, Schatteman G, Wu T, et al. Hypoxia-induced paracrine regulation of vascular endothelial growth factor receptor expression. J Clin Invest. 1996;97:469-476.

59. Waltenberger J, Mayr U, Pentz S, Hombach V. Functional upregulation of the vascular endothelial growth factor receptor KDR by hypoxia. Circulation. 1996;94:1647-1654.

60. Masood R, Cesarman E, Smith DL, Gill PS, Flore O. Human herpesvirus-8-transformed endothelia cells have functionally activated vascular endothelial growth factor/vascular endothelial growth factor receptor. Am J Pathol. 2002;160:23-29.

61. Haslett PA, Hanekom WA, Muller G, Kaplan G. Thalidomide and a thalidomide analogue drug costimulate virus-specific $\mathrm{CD}^{+} \mathrm{T}$ cells in vitro. J Infect Dis. 2003; 187:946-955.

62. Davies FE, Raje N, Hideshima T, et al. Thalidomide and immunomodulatory derivatives augment natural killer cell cytotoxicity in multiple myeloma. Blood. 2001;98:210-216

63. LeBlanc R, Hideshima T, Catley LP, et al. Immu- nomodulatory drug costimulates $T$ cells via the B7-CD28 pathway. Blood. 2004;103:1787-1790.

64. Choi J, Enis DR, Koh KP, Shiao SL, Pober JS. T lymphocyte-endothelial cell interactions. Annu Rev Immunol. 2004;22:683-709.

65. Fonseca R, Barlogie B, Bataille R, et al. Genetics and cytogenetics of multiple myeloma: a workshop report. Cancer Res. 2004;64:1546-1558.

66. Gunsilius E, Duba HC, Petzer AL, et al. Evidence from a leukaemia model for maintenance of vascular endothelium by bone-marrow-derived endothelial cells. Lancet. 2000;355:1688-1691.

67. Barlogie B, Shaughnessy J, Tricot G, et al. Treatment of multiple myeloma. Blood. 2004;103:2032.

68. Bailey AS, Jiang S, Afentoulis M, et al. Transplanted adult hematopoietic stems cells differentiate into functional endothelial cells. Blood. 2004 103:13-19.

69. Sanchez-Elsner T, Botella LM, Velasco B, Corbi A, Attisano L, Bernabeu C. Synergistic cooperation between hypoxia and transforming growth factor- beta pathways on human vascular endothelial growth factor gene expression. J Bio Chem. 2001;276:38527-38535.

70. Derynck R, Zhang Y, Feng XH. Smads: transcriptional activators of TGF-beta responses. Cell. 1998;95:737-740

71. Yang EY, Moses HL. Transforming growth factor beta 1-induced changes in cell migration, proliferation, and angiogenesis in the chicken chorioallantoic membrane. J Cell Biol. 1990;111:731741.

72. Tang B, Vu M, Booker T, et al. TGF-beta switches from tumor suppressor to prometastatic factor in a model of breast cancer progression. J Clin Invest. 2003:112:1116-1124.

73. Kang Y, Siegel PM, Shu W, et al. A multigenic program mediating breast cancer metastasis to bone. Cancer Cell. 2003;3:537-549.

74. Coughlin SR. How the protease thrombin talks to cells. Proc Natl Acad Sci U S A. 1999;96:1102311027.

75. Subramanian SV, Fitzgerald ML, Bernfield M. Regulated shedding of syndecan- 1 and -4 ectodomains by thrombin and growth factor receptor activation. J Biol Chem. 1997;272:1471314720.

76. Seidel C, Sundan A, Hjorth M, et al. Serum syndecan-1: a new independent prognostic marker in multiple myeloma. Blood. 2000;95:388-392.

77. Kaushal V, Kaushal GP, Melkaveri SN, Mehta P. Thalidomide protects endothelial cells from doxorubicin-induced apoptosis but alters cell morphology. J Thromb Haemost. 2004;2:327-334 


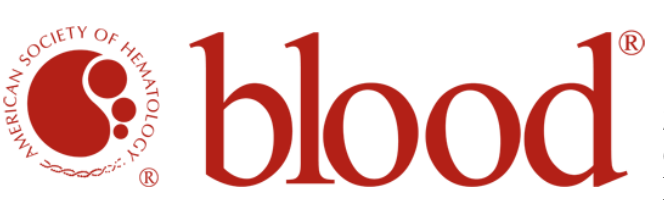

2005 105: 3286-3294

doi:10.1182/blood-2004-06-2101 originally published online December 23, 2004

\section{Circulating endothelial progenitor cells in multiple myeloma: implications and significance}

Hong Zhang, Varsha Vakil, Marc Braunstein, Eric L. P. Smith, Justin Maroney, Laurie Chen, Kezhi Dai, James R. Berenson, M. Mahmood Hussain, Uwe Klueppelberg, Allen J. Norin, Hasan O. Akman, Tayfun Özçelik and Olcay A. Batuman

Updated information and services can be found at:

http://www.bloodjournal.org/content/105/8/3286.full.html

Articles on similar topics can be found in the following Blood collections

Hemostasis, Thrombosis, and Vascular Biology (2485 articles)

Neoplasia (4182 articles)

Information about reproducing this article in parts or in its entirety may be found online at:

http://www.bloodjournal.org/site/misc/rights.xhtml\#repub_requests

Information about ordering reprints may be found online at:

http://www.bloodjournal.org/site/misc/rights.xhtml\#reprints

Information about subscriptions and ASH membership may be found online at:

http://www.bloodjournal.org/site/subscriptions/index.xhtml

Blood (print ISSN 0006-4971, online ISSN 1528-0020), is published weekly by the American Society of Hematology, 2021 L St, NW, Suite 900, Washington DC 20036.

Copyright 2011 by The American Society of Hematology; all rights reserved. 